\title{
Sobre a conexão entre alguns modelos físicos não-lineares
}

\author{
D. X. Andrade, P. H. R. Anjos, P. E. G. Assis* \\ Instituto de Física e Química Universidade Federal de Goiás, Catalão, Goiás, Brazil
}

Recebido em 11 de Abril, 2016. Revisado em 06 de Agosto, 2016. Aceito em 09 de Agosto, 2016.

\begin{abstract}
Neste trabalho revisamos alguns dos sistemas não-lineares mais paradigmáticos e desvendamos algumas das suas surpreendentes interligações. Os problemas de interesse, descrito matematicamente pelas equações de sine-Gordon, Toda e KdV, generalizam modelos físicos conhecidos, como o pêndulo simples, o sistema massa-mola e as ondas lineares em água, respectivamente. Depois de discutirmos as peculiaridades decorrentes da presença de não-linearidades nos modelos, esclarecemos como os sistemas apresentados são relacionados uns aos outros, indicando a existência de uma família de equações que compartilham propriedades como a integrabilidade. Nós mostramos como a equação de KdV pode ser convenientemente discretizada a fim de preservar tais características importantes. Além de apresentarmos as ligações estreitas que esta equação tem com a cadeia de Toda e com a equação sine-Gordon, nós também investigamos outros procedimentos capazes de gerar um sistema integrável discreto a partir do modelo KdV com a discretização de Hirota.
\end{abstract}

Palavras-chave: KdV, Sine-Gordon, Cadeia de Toda.

In this report we review some of the most paradigmatic nonlinear systems and unveil some of their suprising interconnections. The problems of interest, described mathematically by the equations of sine-Gordon, Toda and KdV, generalize well known physical models, the simple pendulum, the mass on a spring and the linear waves, respectively. After discussing the differences arising from the presence of nonlinearities in the models, we clarify how the systems presented are related to each other, indicating existence of a family of equations sharing integrability properties. We show how the KdV equation can be conveniently discretized in order to preserve important properties. Besides presenting the close connections this equation has with respect to the Toda lattice and the sine-Gordon equation, we also investigate other procedures capable of generating a discrete integrable system from the KdV model, as the Hirota discretization.

Keywords: KdV, Sine-Gordon, Toda Lattice.

\section{Introdução}

A maior parte dos fenômenos que ocorrem na natureza envolvem efeitos não-lineares. No entanto, os cursos de graduação tendem a focar nos aspectos lineares destes fenômenos. Dessa forma, em nossa formação, somos levados a pensar que os sistemas não-lineares são tão complicados que nunca podem ser tratados de forma analítica. Assim, pode parecer que o profissional da área deve resignar-se, apenas, a simulações numéricas, como ocorre com a previsão do tempo, por exemplo.

*Endereço de correspondência: paulo.assis@ufg.br
Diante de tal cenário, neste trabalho trazemos uma introdução a alguns problemas não-lineares que podem ser tratados exatamente, com obtenção de soluções analíticas, e que, embora possam parecer à primeira vista completamente independentes, estão relacionados entre si de uma maneira profunda por meio de simetrias. Sabe-se que a presença de simetrias num problema está associada à existência de leis de conservação [1,2], que podem permitir ou facilitar a solução do problema.

Sistemas não-lineares que apresentam um número suficientemente grande de leis de conservação podem ser resolvidos analiticamente e são chamados 
integráveis [3]. O estudo desses sistemas forma uma área de pesquisa que concatena diversos ramos da matemática (como teoria de grupos, álgebra, teoria de representação, topologia, geometria diferencial, sistemas dinâmicos [4]) e permite abordar vários sistemas físicos de relevância atual, como as equações de KdV [5 7], Sine-Gordon [8,9] e Schrodinger nãolinear 10, e, também, os problemas do pêndulo real 11], de Fermi-Pasta-Ulam [12], ondas em águas rasas [13], entre outros. Sendo assim, é uma áea próspera, na física teórica, em especial, no estudo de sistemas compostos por muitas partículas interagentes.

Uma característica comum dos sistemas integráveis é a existência de excitações chamadas sólitons 3.14 , 15, que são pacotes de onda solitários que mantêm sua forma enquanto se propagam, mesmo após sofrer colisões entre si. Descritos pela primeira vez em 1834 [16], os sólitons são manifestações de um balanço sutil entre efeitos dispersivos e não-lineares que, isoladamente, tenderiam a destruir seu perfil de onda com o tempo. Os modelos não-lineares exatamente solúveis, cujo comportamento pode ser completamente descrito em qualquer instante, por apresentar soluções em forma fechada, servem de laboratório para a aplicação de métodos analíticos e perturbativos, e também como ponto de partida para o estudo de sistemas para os quais não há soluções analíticas e as simulações numéricas são computacionalmente custosas, quando não proibitivas.

A existência de ondas cujo comportamento pode ser interpretado como partículas é mais que uma curiosidade e possui implicações e aplicações relevantes. Os sólitons podem ser usados para modelar algumas partículas exóticas 17 ou para descrever o comportamento do encontro das correntes fluvias do Rio Amazonas com as águas oceânicas durante as marés altas, que geram o fenômeno conhecido como pororoca [18. De um ponto de vista matemático, os sólitons permitem obter soluções exatas de equações diferenciais não lineares que, a partida, poderiam ser apenas investigadas numericamente.

Diante do exposto, neste trabalho, revisamos alguns dos mais paradigmáticos sistemas não-lineares integráveis e desvendamos algumas de suas supreendentes interconexões, num contexto acessível a estudantes de graduação, com algum domínio de Mecânica Clśsica e Equações Diferenciais. Em particular, estudamos sistemas bem conhecidos, como o pêndulo simples, o sistema massa-mola e a equação de onda linear, para mostrar que eles são, na verdade, limites lineares das equações de sine-Gordon, Toda e KdV, respectivamente. Mostramos, de forma clara, como esses modelos, aparentemente distintos, estão relacionados, sendo, efetivamente, representações distintas de simetrias escondidas.

Tendo em vista nosso objetivo pedagógico, organizamos este trabalho da seguinte forma: Iniciamos, na seção 2, com uma breve introdução a sistemas não-lineares. Em particular, calculamos a solução do pêndulo para além da tradicional aproximação de pequenas oscilações e usamos esses resultados para introduzir o modelo de Sine-Gordon em 3 . Em seguida, na seção 4 introduzimos rapidamente o problema Fermi-Pasta-Ulan (FPU) e a modificação proposta por Toda [12]. Com essas construções, mostramos, na subseção 4.2, por que o modelo de SineGordon pode ser entendido como uma teoria de campo de Toda. Posteriormente, a equação KdV é discutida na seção 5 , onde mostramos como ela pode ser entendida como o limite ultravioleta da cadeia de Toda. Ademais, em 5.2 , discutimos uma descrição alternativa da equação de $\mathrm{KdV}$ por meio do operador de Hirota, que nos permite construir a solução para multi-sólitons. Como uma aplicação dessa discretização, em 5.3 . construimos a solução para um sóiton. Por fim, discutimos, subseção 5.4 . rapidamente como as equações KdV e Sine-Gordon estão conectadas. E, na seção 6 apresentamos nossas conclusões.

\section{Sistemas Lineares e Não-Lineares}

Embora as equações lineares sejam geralmente mais simples de se resolver, a não-linearidade manifestase em variados sistemas físicos. Por este motivo existe um crescente interesse físico e matemático para analisar os fenômenos não-lineares.

Um sistema físico descrito por uma equação diferencial é linear quando, para ela, é válido o princípio da superposição [19]. Além do mais, a resposta a pequenas variações em seus parâmetros iniciais, ou a estímulos externos, é usualmente suave e diretamente proporcional ao estímulo. Em geral, um pulso sob uma dinâmica linear normalmente espalhar-seão com o tempo, num efeito chamado de dispersão. Ele é o responsável por fazer as ondas perderem sua forma e desaparecer. 
Já para o caso não-linear, este princípio deixa de ser verdadeiro, ou seja, duas soluções de uma equação não-linear não podem ser combinadas linearmente para formar outra solução. Isso dificulta a obtenção de métodos analíticos gerais para resolver equações não-lineares. A resposta a estímulos externos não é linearmente proporcional ao estímulo, diferentemente do que ocorre nos sistemas lineare. Assim, uma pequena variação nos parâmetros do sistema pode provocar uma enorme diferença no movimento. Uma outra importante propriedade das excitações não-lineares é que podem apresentar-se na forma de estruturas altamente coerentes e localizadas como os sólitons, que viajam a velocidade constante e mantêm a forma por longas distâncias.

Uma sistema físico que ilustra claramente tais diferenças é o clássico pêndulo simples. O movimento de um pêndulo simples em pequenas oscilações é harmônico e descrito por uma equação linear. Já o regime de grandes oscilações é descrito por uma equação não-linear. Enquanto a solução para o pêndulo linear é bastante simples, encontrar soluções exatas para ângulos não necessariamente pequenos (pêndulo não-linear) é consideravelmente mais difícil.

\subsection{O pêndulo real}

O estudo do pêndulo não-linear é oportuno para mostrar que fenômenos complexos podem ser originados por modelos bastantes simples, desde que tratados por meio de uma abordagem não-perturbativa. Podemos resolver o problema do pêndulo não-linear a partir da conservação da energia mecânica. Dado que a energia potencial associada ao problema é

$$
U(\theta)=m g l(1-\cos \theta),
$$

podemos escrever a equação característica do movimento pendular na forma

$$
m l^{2} \ddot{\theta}+m g l \sin \theta=m l^{2} \ddot{\theta}+U^{\prime}(\theta)=0 .
$$

Para resolvê-la multiplicamos ambos os lados de (1) por $\dot{\theta}$,

$$
m l^{2} \ddot{\theta} \dot{\theta}+m g l \sin \theta \dot{\theta}=m l^{2} \ddot{\theta}+U^{\prime}(\theta) \dot{\theta}=0,
$$

para obter,

$$
\frac{d}{d t}\left(\frac{m l^{2}}{2} \dot{\theta}^{2}+U(\theta)\right)=0,
$$

que é a familiar lei de conservação da energia,

$$
\frac{m l^{2}}{2} \dot{\theta}^{2}+U(\theta)=\frac{m l^{2}}{2} \dot{\theta}_{\max }^{2}+U\left(\theta_{\max }\right)=E_{\max } .
$$

Percebe-se que, no nosso caso, no ângulo de máxima abertura $\theta=\theta_{\max }= \pm \pi$ a velocidade se anula e temos $\dot{\theta}=0$, fixando-se dessa forma a energia para completarmos uma volta. Assim, ao reescrevermos a equação (2),

$$
\left(\frac{d \theta}{d t}\right)^{2}=2 \omega^{2}(1+\cos \theta),
$$

notamos que ela nos permite escrever a relação

$$
\frac{1}{\sqrt{2}} \int \frac{d \theta}{\sqrt{1+\cos \theta}}=\omega \int d t=\omega\left(t-t_{0}\right)=\omega \tau,
$$

para a variação da posição angular com variações infinitesimais de tempo.

Na usual aproximação de ângulos pequenos, para a qual se considera $\theta \ll 1$, o lado esquerdo da expressã acima produz $\int \frac{1}{\sqrt{4-\theta^{2}}} d \theta=\arcsin \left(\frac{\theta}{2}\right)$, indicando que de fato o ângulo varia harmonicamente nesse regime.

No caso de uma amplitude genérica, uma forma de simplificar a obtenção da solução é notar que

$$
\frac{d}{d \phi} \log \left[\tan \left(\frac{\phi}{4}\right)\right]=\frac{1}{\sqrt{2}} \frac{1}{\sqrt{1-\cos \phi}} .
$$

Sendo assim, usando-se $\theta=\phi+\pi$, chegamos à solução,

$$
\omega \tau=\frac{1}{\sqrt{2}} \int \frac{d \theta}{\sqrt{1+\cos \theta}}=\log \left(\tan \frac{\theta}{4}\right)
$$

a partir da qual podemos isolar a expressão para a posição angular em função do tempo,

$$
\theta(t)=4 \arctan e^{\omega\left(t-t_{0}\right)},
$$

representada na Figura 1, tracejado verde. Com isso, a solução que procuramos, pode ser escrita como,

$$
\theta(t)=4 \arctan \left(\frac{e^{\omega\left(t-t_{0}\right)}-1}{e^{\omega\left(t-t_{0}\right)}+1}\right),
$$

cuja representação gráfica é dada pela Figura 1. tracejado azul.

Nota-se que a posição angular não varia linearmente; na verdade, como esperado, temos uma variação aproximadamente linear nas regiões próximas da origem, isto é, quando as aberturas angulares 


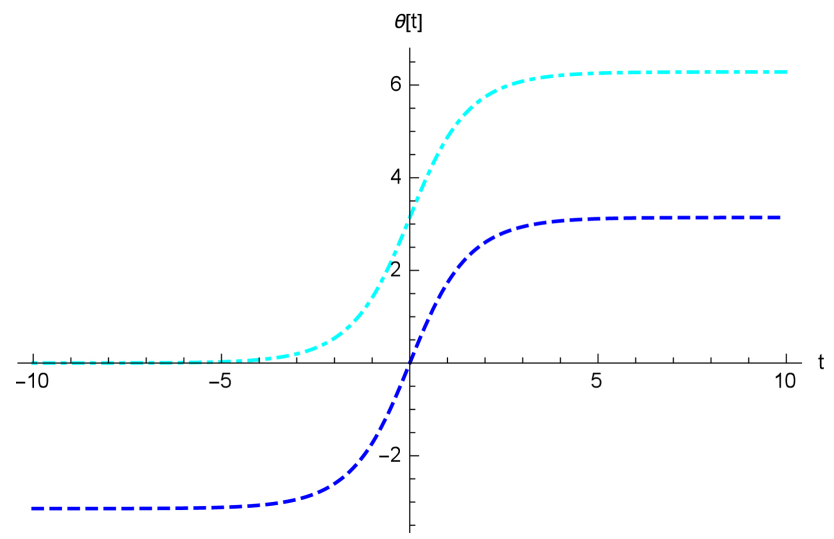

Figura 1: Representações gráficas das expressões (3) e (4), indicando a posição do pêndulo não linear em função do tempo.

são pequenas. Percebe-se, contudo, que, conforme o pêndulo gira por múltiplos de $\pi$ a partir da origem, ele atinge um ponto de equilíbrio (instável) e o ângulo poderia parar de variar.

Tal afirmação pode ser verificada na fig. 2, onde sobrepomos o comportamento do pêndulo simples, em cinza, com o do pêndulo real, não-linear, em azul, e observamos como as soluções são aproximadamente equivalentes apenas para pequenas amplitudes. Conforme aumentamos a amplitude, chega um ponto em que ao aumentarmos o deslocamento relativo do pêndulo o torque restaurador já não cresce na mesma proporção. De fato, o torque é descrito matematicamente por uma função senoidal.

Uma forma de analisar o comportamento futuro do pêndulo n ao-linear é utilizar a ideia do espaço de fase, mostrado na Figura 3 , representado pelas variáveis dinâmicas do sistema, a posição $\theta$ e a velocidade angular $\dot{\theta}$. Nela, percebe-se que as curvas fechadas representam oscilações com energia potencial $(E<2 m g l)$ e são, portanto, periódicas e limitadas, não podendo assumir qualquer ângulo. No caso das curvas abertas $(E>2 m g l)$ temos um movimento que não estão limitado, ou seja, o pêndulo pode girar de 0 a $2 \theta$ tanto no sentido horário quanto antihorário e continuar o movimento indefinidamente. A regularidade nos sistemas integráveis é notável, não estando presente em outros regimes não lineares, como o caótico, no qual as curvas tenderiam a preencher todo o espaço de fase de forma irregular.

\section{O pêndulo real e Sine-Gordon estático}

Um sistema de pêndulos enfileirados e acoplados por molas de torção pode ser descrito pela equação
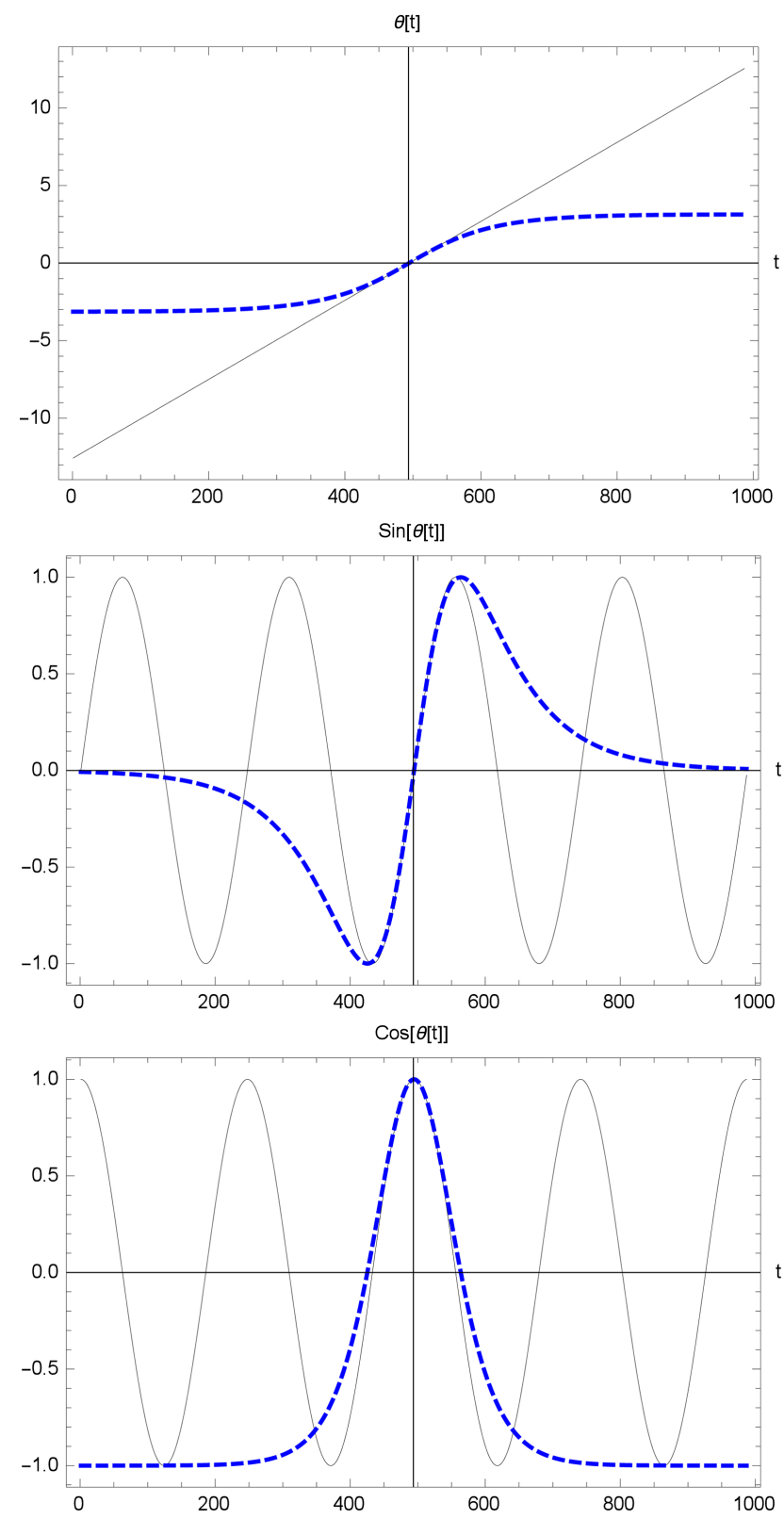

Figura 2: Nas figuras acima mostramos uma comparação entre o comportamento linear, indicado pelas linhas contínuas acinzentadas, e o comportamento solitónico, representado pelas linhas tracejadas azuis. Na parte superior tem-se o deslocamento angular do pêndulo em função do tempo. No gráfico intermediário mostramos como se comporta a força sentida pelo pêndulo devido a sua abertura angular que varia no tempo. Finalmente, na figura inferior indicamos o potencial sentido pelo corpo.

de Sine - Gordon. Conhecida desde o séc XIX, no entanto, ganhou importância apenas nos anos de 1970 quando se percebeu que sua solução levava a estrutura do tipo sólitons, "kink" e "antikink", como mostrado na Figura 4. A equação de Sine-Gordon é uma equação diferencial parcial não linear que 


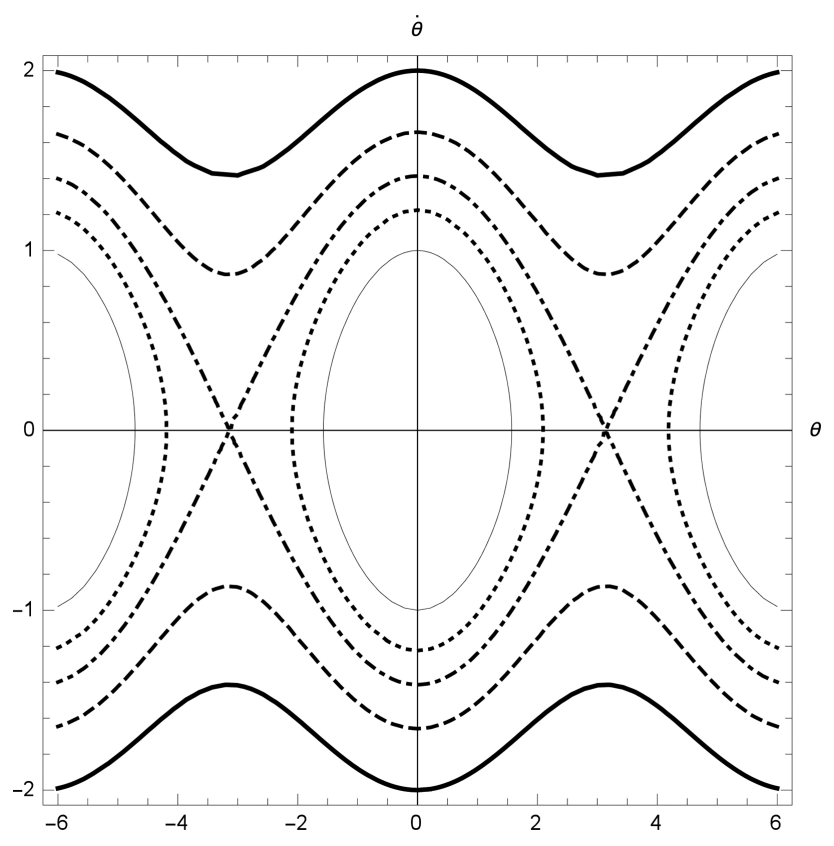

Figura 3: Trajetórias no espaço de fase para o pêndulo real com energias $E=\frac{m g l}{100}$ (curva contínua fina), $E=m g l$ (curva pontilhada), $E=2 m g l$ (curva tracejada-pontilhada), $E=4 m g l$ (curva tracejada), $E=6 m g l$ (curva contínua grossa).

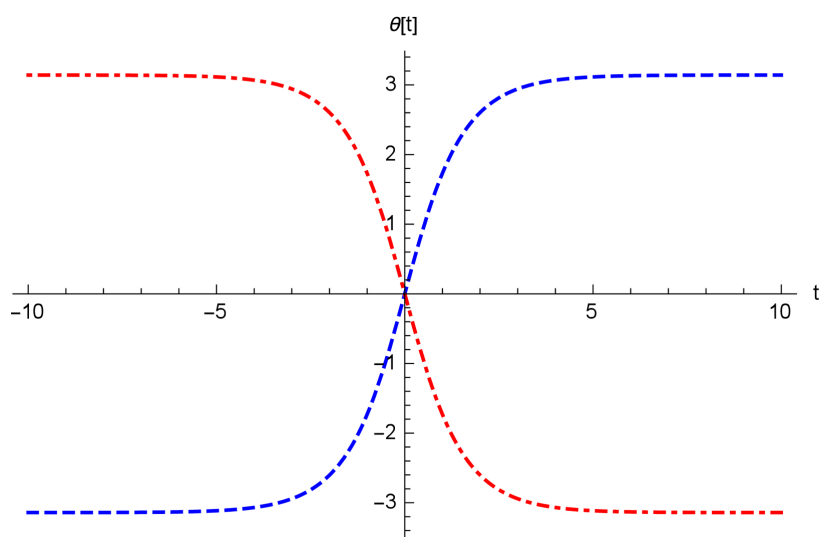

Figura 4: Soluções do tipo kink $(\epsilon=+1)$, indicada pela curva ascendente tracejada em azul, e do tipo anti-kink $(\epsilon=-1)$, mostrada na curva descendente pontilhada em vermelho.

envolve o operador de d'Alembert e o seno de uma função, da seguinte forma

$$
\phi_{t t}+\phi_{x x}+\frac{\mu^{2}}{\beta} \sin \beta \phi=0,
$$

onde $\phi=\phi(x, t)$ é uma função do espaço e do tempo. Ela aparece em aplicações físicas que vão desde teorias de campos relativísticos e junções Josephson até linhas de transmissões mecânicas.

No caso do pêndulo, $\phi(x, t)$ descreve o seu ângulo de rotacão. Note que, para pequenas amplitudes $(\sin \phi \simeq \phi)$ a equação (5) se reduz a

$$
\phi_{t t}+\phi_{x x}+\mu^{2} \phi=0
$$

conhecida como equação de Klein-Gordon e admite soluções da forma

$$
\phi(x, t)=\phi_{0} \cos (k x-w t) \quad \omega=\sqrt{\mu^{2}+k^{2}} .
$$

No entanto, aqui, estamos interessados nos casos em que o pêndulo pode oscilar em amplitudes arbitrárias, pequenas ou grandes. Note que os papéis de $x$ e $t$, nessa equação, são intercambiáveis e podemos inicialmente supor que $\phi_{t t}=0$, de modo que não há variação de $\phi$ no tempo, no chamado regime estático. Assim, temos a equação para o pêndulo não linear,

$$
\phi_{x x}+\frac{\mu^{2}}{\beta} \sin \beta \phi=0
$$

cuja solução,

$$
\beta \phi(x)=\frac{4}{\beta} \arctan \left(\frac{e^{ \pm \mu x}-1}{e^{ \pm \mu x}+1}\right),
$$

já foi demonstrada na seção (2). Como a equação (5) é invariante por transformações de Lorentz podemos aplicar um boost,

$$
x \rightarrow \cosh (\chi)[x-c \tanh (\chi) t],
$$

na equação (7) para obter a solução viajante da equação de Sine-Gordon (5),

$$
\phi(x, t)=\frac{4}{\beta} \arctan \left(\frac{e^{\epsilon \mu(\cosh (\chi) x-\sinh (\chi) c t)}-1}{e^{\epsilon \mu(\cosh (\chi) x-\sinh (\chi) c t)}+1}\right) .
$$

A solução acima representa uma onda solitária localizada viajando com uma velocidade $c$. De acordo com os sinais $\epsilon= \pm$ classificamos a solução em kink, o pêndulo rotaciona de 0 para $2 \pi$, e antikink,o pêndulo rotaciona de 0 para $-2 \pi$, como representado na Figura (4), abaixo.

O que acabamos de ver foi um problema envolvendo uma equação diferencial não-linear para o qual foi possível encontrar uma solução exata, isto é, um problema integrável. A seguir veremos um pouco sobre os primórdios dessa área e apresentaremos alguns outros exemplos de modelos exatamente integráveis. 


\section{Cadeias de Toda e o Problema de Fermi-Pasta-Ulam}

As pesquisas entorno dos sólitons ficaram inertes até o início de 1950, quando o estudo de oscilações em redes não lineares ganhou evidência, após os trabalhos de Fermi, Pasta e Ulam no problema da partição de energia 20]. O problema de Fermi-Pasta Ulam foi um dos precursores das simulações computacionais na área da Física e constitui-se de simulações em uma rede de partículas ligadas entre si através de molas que obedecem a Lei de Hooke com uma correção não-linear quadrática. Esperava-se que a deformação linear quebrasse o comportamento ergódico do sistema, mas, surpreendentemente, os estados visitados durante a evolução não eram quaisquer. A distribuição de energia entre os modos apresentava um comportamento aproximadamente periódico, fato atribuído à presença de simetrias escondidas. Em 1981, Morikazu Toda modificou o problema de FPU introduzindo uma interação exponencial, eq. (8), entre os elementos da cadeia, tornando-o um problema integrável 12 .

Como citado o problema de Fermi-Pasta Ulam consiste em modificar a rede linear, adicionando uma não linearidade quadrática $\left(\alpha k \Delta x^{2}\right)$ à força de Hooke, da seguinte forma,

$$
F=-k \Delta x-\alpha k \Delta x^{2}
$$

onde $\alpha$ é o parâmetro de deformação (arbitrariamente grande ou pequeno) e $\Delta x$ é o deslocamento em relação ao ponto de equilíbrio. Logo, a força experimentada por uma partícula j na rede de FPU permite-nos escrever a equação de movimento satisfeita por

$m \ddot{x}_{j}=k\left(x_{j+1}-2 x_{j}+x_{j-1}\right)\left[1+\alpha\left(x_{j+1}-x_{j-1}\right)\right]$.

O FPU não é um sistema integrável: o número de quantidades conservadas é insuficiente para restringir univocamente sua evolução. A suficiência das simetrias em uma cadeia não-linear aparece nos modelos de Toda, como veremos a seguir.

\subsection{A cadeia de Toda}

Os estudos das redes não-lineares de Fermi, Pasta e Ulam mostraram que as mesmas possuem comportamento aproximadamente periódico [20]. Toda, em seu trabalho, concluiu que estas estruturas estariam ligadas a uma interação exponencial entre as partículas da rede 21]. E mostrou que esta interação admite ondas periódicas, o que estava de acordo com os estudos de Fermi,Pasta e Ulam.

A rede de Toda é constituída de $N$ corpos discretos sujeitos a uma interação que cresce exponcialmente com a distâcia entre os corpos vizinhos, de modo que o par $q_{n}, q_{n-1}$ está associado a um potencial

$$
V\left(q_{n}, q_{n-1}\right)=\frac{\mu^{2}}{4 \beta^{2}} e^{\beta\left(q_{n}-q_{n-1}\right)}
$$

e, portanto, a $n$-ésima partícula sofre uma interação devida ao seu vizinho à esquerda e também uma devida ao seu vizinho da direita,

$$
V_{n}=\frac{\mu^{2}}{4 \beta^{2}}\left(e^{\beta\left(q_{n}-q_{n-1}\right)}+e^{\beta\left(q_{n+1}-q_{n}\right)}\right) .
$$

Para cada corpo discreto presente na rede, tem-se uma amplitude $y_{n}$, tal que $\dot{y}_{n}$ e $\ddot{y}_{n}$ correspondem à velocidade e à aceleração do corpo que ocupa o n-ésimo sítio da rede. No caso de massa unitária, as equações de movimento satisfeitas pelas partículas de sua rede podem ser obtidas pela função Hamiltoniana associada ao problema,

$$
H=\sum_{k=1}^{N}\left(\frac{1}{2} p_{k}^{2}+V_{k}\right)
$$

descritas por

$$
\begin{aligned}
\dot{q_{n}} & =\left\{q_{n}, H\right\}_{P B}=+\frac{\partial H}{\partial p_{n}}=p_{n} \\
\dot{p_{n}} & =\left\{p_{n}, H\right\}_{P B}=-\frac{\partial H}{\partial q_{n}}=-\frac{\partial V}{\partial q_{n}}=F_{n} .
\end{aligned}
$$

Logo, temos um sistema acoplado regido por

$$
\ddot{q_{n}}=\dot{p}_{n}=F_{n}=\frac{\mu^{2}}{4 \beta}\left(e^{\beta\left(q_{n}-q_{n-1}\right)}-e^{\beta\left(q_{n+1}-q_{n}\right)}\right) .
$$

\subsection{O modelo de Sine-Gordon como uma teoria de campo de Toda}

O caso mais simples, não trivial, correspondente a apenas dois corpos, com $N=2$, interagindo de acordo com o potencial de Toda é,

$$
\begin{aligned}
& \ddot{q}_{1}=\frac{\mu^{2}}{4 \beta}\left(e^{\beta\left(q_{1}-q_{0}\right)}-e^{\beta\left(q_{2}-q_{1}\right)}\right), \\
& \ddot{q}_{2}=\frac{\mu^{2}}{4 \beta}\left(e^{\beta\left(q_{2}-q_{1}\right)}-e^{\beta\left(q_{3}-q_{2}\right)}\right),
\end{aligned}
$$


onde consideramos por simplicidade o problema com condições de contorno periódicas de modo que devemos interpretar $q_{0}=q_{2}$ e $q_{3}=q_{1} \mathrm{e}$

$$
\ddot{q}_{1}-\ddot{q}_{2}=\frac{\mu^{2}}{2 \beta}\left(e^{\beta\left(q_{1}-q_{2}\right)}-e^{\beta\left(q_{2}-q_{1}\right)}\right) .
$$

Introduzindo $q=q_{1}-q_{2}$ obtemos simplesmente

$$
\ddot{q}=\frac{\mu^{2}}{2 \beta}\left(e^{\beta q}-e^{-\beta q}\right)=\frac{\mu^{2}}{\beta} \sinh (\beta q) .
$$

No caso de uma constante de acoplamento imaginária $\beta=i b$, teremos

$$
\ddot{q}=\frac{\mu^{2}}{2 \beta}\left(e^{i b q}-e^{-i b q}\right)=-\frac{\mu^{2}}{2 b} \sin (b q) .
$$

Poderíamos considerar também o potencial de Toda associado a um campo, ao invés de partículas, $q_{n}(t) \rightarrow \phi_{n}(x, t)$ e $p_{n}(t) \rightarrow \pi_{n}(x, t)$, a partir da seguinte densidade Hamiltoniana

$\mathcal{H}=\sum_{k=1}^{N}\left[\frac{1}{2} \pi_{k}^{2}+\frac{\mu^{2}}{4 \beta^{2}}\left(e^{\beta\left(\phi_{k}-\phi_{k-1}\right)}+e^{\beta\left(\phi_{k+1}-\phi_{k}\right)}\right)\right]$

As equações de movimento são dadas por

$$
\begin{aligned}
\frac{\partial}{\partial t} \phi_{n} & =\left\{\phi_{n}, \mathcal{H}\right\}_{P B}=\int d y \delta(x-y) \frac{\delta \mathcal{H}}{\delta \pi_{n}[y]}=\pi_{n}, \\
\frac{\partial}{\partial t} \pi_{n} & =\left\{\pi_{n}, \mathcal{H}\right\}_{P B}=\int d y \delta(x-y) \frac{\delta \mathcal{H}}{\delta \varphi_{n}[y]}, \\
\frac{\partial^{2}}{\partial t^{2}} \phi_{n} & =\frac{\partial^{2}}{\partial x^{2}} \phi_{n}+\frac{\mu^{2}}{\beta}\left(e^{\beta\left(\phi_{n}-\phi_{n-1}\right)}-e^{\beta\left(\phi_{n+1}-\phi_{n}\right)}\right)
\end{aligned}
$$

de modo que, para $N=2$ com condições de contorno periódicas e $i \phi=\phi_{1}-\phi_{2}$, temos

$$
\phi_{t t}=\phi_{x x}+\frac{\mu^{2}}{\beta} \sin (\beta \phi),
$$

que é a equação de sine-Gordon.

\section{A equação de $K d V$ para fluidos rasos}

A derivação da equação de onda linear é baseada em três suposições simplificadoras: (i) Não há dissipação, ou seja, a equação é invariante com a inversão do tempo; (ii) Não há dispersão, ou seja, a velocidade de grupo, $v_{g}=\frac{\partial \omega}{\partial k}=v$ é constante; (iii) A amplitude de oscilação é pequena e, então, os termos não lineares são omitidos. Iniciando pela solução mais conhecida para uma onda viajante,

$$
\varphi(x, t)=\varphi_{0} \cos (k x+\omega t)
$$

sendo $k$ o número de onda dado por $k=\frac{2 \pi}{\lambda}$ e $\omega=\frac{2 \pi}{T}$ a frequência angular, temos

$$
\frac{\partial^{2} \varphi}{\partial x^{2}}-\frac{1}{v^{2}} \frac{\partial^{2} \varphi}{\partial t^{2}}=0
$$

conhecida como equação de onda linear. Ou seja, a equação (9) é na verdade uma das soluções que satisfaz a equação de onda linear. A solução geral da equação de onda linear (10) é a superposição de duas ondas viajando em direções opostas,

$$
\varphi(x, t)=f(x-v t)+g(x+v t),
$$

ou seja, uma onda viajando para a direita e outra viajando para a esquerda, sendo $f$ e $g$ funções arbitrárias. Cada uma dessas duas ondas é solução de 10.

Com o intuito de modificarmos a relação de dispersão, $\omega(k)=k v$, uma das alterações mais imediatas a se fazer consiste na introdução de um termo dispersivo, substituindo-a por

$$
\omega(k)=\left(k-B k^{3}+\cdots\right) v .
$$

Ao considerar pequena a dispersão introduzida, podemos manter apenas os dois primeiros termos de (12) e a solução da equação de onda em sua forma complexa pode ser expressa como

$$
\varphi(x, t)=\varphi_{0} e^{i\left(k x-\left(k-\beta k^{3}\right) v t\right) .}
$$

Onde, então, se verifica que a equação satisfeita por essa onda tem a forma

$$
\frac{\partial \varphi}{\partial x}+B \frac{\partial^{3} \varphi}{\partial x^{3}}+\frac{1}{v} \frac{\partial \varphi}{\partial t}=0
$$

e pode, convenientemente, ser reescrita na forma de uma lei de conservação, em termos da equação de continuidade,

$$
\frac{\partial \rho}{\partial t}+\frac{\partial J}{\partial x}=0
$$

onde temos, por comparação,

$$
\rho=\frac{\varphi}{v}, \quad \text { e } \quad J=\varphi+B \frac{\partial^{2} \varphi}{\partial x^{2}} .
$$

Por outro lado, a fim de introduzir efeitos de não linearidade, adicionamos em $J$, acima, um termo quadrático em $\phi$,

$$
J=\varphi+B \frac{\partial^{2} \varphi}{\partial x^{2}}+\frac{A}{2} \varphi^{2},
$$

Revista Brasileira de Ensino de Física, vol. 39, nº 1, e1307, 2017 
de modo que a equação resultante regendo esse comportamento assume a forma,

$$
\frac{\partial \varphi}{\partial x}+B \frac{\partial^{3} \varphi}{\partial x^{3}}+A \frac{\partial \varphi}{\partial x}+\frac{1}{v} \frac{\partial \varphi}{\partial t}=0 .
$$

Ao realizar uma mudança de variáveis e reescalar $\phi$, conforme segue,

$$
\begin{aligned}
& \varphi \rightarrow u=k_{0}+k_{1} \varphi \\
& x \rightarrow \xi=k_{2}+k_{3} x, \\
& t \rightarrow \tau=k_{4}+k_{5} t,
\end{aligned}
$$

obtêmos relações que permitem reescrever (13) como

$$
\frac{\partial u}{\partial \tau}+\frac{v}{k_{5}}\left[\left(\frac{A u}{k_{1}}-\frac{A k_{0}}{k_{1}}+1\right) k_{3} \frac{\partial u}{\partial \xi}+B k_{3}^{3} \frac{\partial^{3} u}{\partial \xi^{3}}\right]=0
$$

ou ainda, de forma mais concisa, com $k_{0}=\frac{1}{A}, k_{1}=$ $k_{3}=1$ e $k_{5}=v$,

$$
u_{\tau}+\left(A \frac{u^{2}}{2}+B u_{\xi \xi}\right)_{\xi}=0
$$

equivalente à conhecida equação de $\mathrm{KdV}$,

$$
u_{t}+A u u_{x}+B u_{x x x}=0 .
$$

Logo, vê-se que tal equação pode ser vista como uma generalização relativamente natural da equação de onda de D'Alembert para a qual foram introduzidas deformações simples capazes de gerar efeitos dispersivos e não lineares.

\subsection{A equação de $\mathrm{KdV}$ como o limite contínuo para a cadeia de Toda}

Ambas, a equação de KdV para ondas rasas e a rede discreta não linear de Toda, têm soluções multisolitônicas. Suas soluções e métodos de soluções são similares. Nesse seção iremos descrever a relação entre elas iniciando da cadeia de Toda para chegar na equação de $\mathrm{KdV}$, mantendo a propriedade de ser integrável. O objetivo é alcançado ao realizar a aproximação da cadeia de Toda para o limite contínuo.

Iniciando pela equação de Newton para uma partícula sujeita a um potencial $V(\varphi)$, responsável pela interação entre vizinhos, que depende da distância entre as partículas vizinhas, sendo que as duas primeiras vizinhas exercem forças em sentidos opostos, temos

$$
m \frac{\partial^{2} y_{n}}{\partial t^{2}}=-V^{\prime}\left(y_{n}-y_{n-1}\right)+V^{\prime}\left(y_{n+1}-y_{n}\right) .
$$

Podemos supor que a interação pode ser expandida em termos de um deslocamento relativo $r_{n}=$ $y_{n+1}-y_{n}$ de uma forma que generaliza o potencial de Hooke para uma força elástica linear, agora incluindo termos não lineares,

$$
V\left(r_{n}\right)=\frac{1}{2} k r_{n}^{2}+\frac{1}{3} k a_{0} r_{n}^{3}+\frac{1}{4} k a_{1} r_{n}^{4}+\cdots .
$$

É possível considerarmos que a amplitude da onda discreta $y_{n}(t)$ varia tão suavemente que pode ser considerada uma função contínua de $x=n h$, sendo $h$ a distância média entre duas partículas adjacentes. Dessa forma, temos uma discretização do perfil da onda, $y(x, t) \rightarrow y_{n}(t)$.

Para caracterizar a amplitude da onda num sítio vizinho da rede discreta podemos também utilizar uma expansão de Taylor,

$y_{n \pm 1}=y \pm h \frac{\partial y}{\partial x}+\frac{h^{2}}{2 !} \frac{\partial^{2} y}{\partial x^{2}} \pm \frac{h^{3}}{3 !} \frac{\partial^{3} y}{\partial x^{3}}+\frac{h^{4}}{4 !} \frac{\partial^{4} y}{\partial x^{4}} \pm \cdots$,

de modo que a equação de movimento pode ser escrita, com $c_{0}=h \sqrt{\frac{k}{m}}, \epsilon=2 a_{0} h, h^{2} \epsilon \rightarrow 0$, como

$$
\frac{1}{c_{0}^{2}} \frac{\partial^{2} y}{\partial t^{2}}=\frac{\partial^{2} y}{\partial x^{2}}+\epsilon \frac{\partial y}{\partial x} \frac{\partial^{2} y}{\partial x^{2}}+\frac{h^{2}}{12} \frac{\partial^{4} y}{\partial x^{4}}
$$

similar àquela conhecida como equação de Boussinesq [13], descrevendo ondas em águas razas, sendo, em princípio, exatamente integrável.

Se usarmos uma lenta variação na escala do tempo com $u=\frac{\partial y}{\partial \xi}, \quad \tau=\frac{1}{2} \epsilon c_{0} t$, e $\xi=x-c_{0} t$, temos, desprezando termos de ordem $\epsilon^{2}$,

$$
\epsilon u_{\tau}+\epsilon u u_{\xi}+\frac{h^{2}}{12} u_{\xi \xi \xi}=0,
$$

de modo que ao assumirmos que $|\epsilon| \sim h^{2}$ obtemos a integrável equação da $\mathrm{KdV}$,

$$
u_{\tau}+u u_{\xi}+\frac{1}{12} u_{\xi \xi \xi}=0 .
$$

Existe ainda uma maneira alternativa de transformar a equação de Toda, em sua forma exponencial, sem expansões, na equaç ao de KdV. Para realizar tal transformação nós primeiramente escrevemos a equação de movimento da cadeia de Toda em termos da variável $r_{n}=y_{n+1}-y_{n}$,

$$
\frac{\partial^{2} r_{n}}{\partial t^{2}}=2 e^{-r_{n}}-e^{-r_{n-1}}-e^{-r_{n+1}},
$$


e utilizando a força entre duas partículas como sendo igual a $f_{n}=e^{-r_{n}}-1$, a equação de movimento expressa-se da seguinte maneira,

$$
\frac{\partial^{2}}{\partial t^{2}} \log \left(f_{n}+1\right)=f_{n+1}+f_{n-1}-2 f_{n} .
$$

Finalmente, introduzimos um parâmetro positivo $h$, tal que $0<h \leq 1$, e reescalamos o parâmetro temporal bem como a variável de amplitude da onda discreta de acordo com $t=\frac{\tau}{h^{3}}$, e $f_{n}=C h^{2} u_{n}(\tau)$, levando-nos a

$$
\frac{\partial^{2}}{\partial \tau^{2}} \log \left(C h^{2} u_{n}+1\right)=\frac{C}{h^{4}}\left(u_{n+1}+u_{n-1}-2 u_{n}\right) .
$$

Podemos similarmente reescalar o parâmetro espacial do problema em termos do parâmetro adimensional $h$ de tal modo que nos encontremos num referencial em movimento para a direita com velocidade $\left(\frac{1}{h^{2}}-h^{2}\right)>0$, tal que $x=n h-\left(\frac{1}{h^{2}}-h^{2}\right) \delta \tau$, e a amplitude $u_{n}$ pode ser vista como uma função das variáveis $x$ e $\tau, u(x, \tau)=u_{n}(\tau)$. Em termos delas, a equação para a cadeia exponencial de Toda na forma (15) é reescrita como

$$
\begin{gathered}
\left(\delta \frac{\partial}{\partial \tau}-\left(\frac{1}{h^{2}}-h^{2}\right) \frac{\partial}{\partial x}\right)^{2} \log \left(1+C h^{2} u(x, \tau)\right) \\
\quad=\frac{C}{h^{4}}[u(x+h, \tau)+u(x-h, \tau)-2 u(x, \tau)] .
\end{gathered}
$$

Ao substituirmos $h=1$ na equação acima, obtemos novamente a equação original exponencial de Toda. Também quando $h \neq 1$, ainda temos a cadeia exponencial de Toda, porque nós apenas mudamos as escalas e adicionamos coordenadas de movimento. No entanto, temos que considerar o caso no limite em que $h=0$. No limite em (16) quando $h$ tende a 0 pelo lado positivo, $h \rightarrow 0_{+}$, o lado esquerdo da equação 16 torna-se,

$$
-2 \delta C \frac{\partial^{2} u}{\partial x \partial \tau}+\frac{C}{h^{2}} \frac{\partial^{2} u}{\partial x^{2}}-\frac{C^{2}}{2} \frac{\partial^{2} u}{\partial x^{2}} .
$$

após usarmos expansão em série de Taylor para a função logarítimica. Já o lado direito da equação (16) se torna,

$$
\frac{C}{h^{2}} \frac{\partial^{2} u}{\partial x^{2}}+\frac{C}{12} \frac{\partial^{4} u}{\partial x^{4}} .
$$

Igualando (17) e (18) para formar (16) temos, após o cancelamento dos termos semelhantes,

$$
-\frac{\partial}{\partial x}\left(\frac{\partial u}{\partial \tau}+\frac{C}{4 \delta} \frac{\partial u}{\partial x}\right)=\frac{1}{24 \delta} \frac{\partial^{4} u}{\partial x^{4}} .
$$

Tomando $\delta=\frac{1}{24 B}$ e reescalando, convenientemente, o parâmetro $C$ da seguinte forma, $C \rightarrow \frac{A B}{12}$, temos

$$
-\frac{\partial}{\partial x}\left(\frac{\partial u}{\partial \tau}+\frac{A}{2} \frac{\partial u}{\partial x}\right)-B \frac{\partial^{4} u}{\partial x^{4}}=0
$$

ou ainda,

$$
\frac{\partial}{\partial x}\left(u_{\tau}+A u u_{x}+B u_{x x x}\right)=0 .
$$

Por fim, usando-se a condição de que quando $x$ tende ao infinito a amplitude $u$ deve anular-se, nós obtemos a equação de $\mathrm{KdV}$,

$$
u_{\tau}+A u u_{x}+B u_{x x x}=0 .
$$

Essa é portanto uma maneira alternativa de escrever a equação de Korteweg e de Vries para as ondas solitárias em canais como o limite contínuo do modelo de Toda para uma cadeia discreta de partículas que interagem por meio de uma generalização dos potenciais de Hooke e Fermi-Pasta-Ulam.

\subsection{A forma bilinear de Hirota para a $\mathrm{KdV}$}

Nesta seção apresentamos um outro procedimento, devido a Hirota [22], que produz uma nova forma para a equação de KdV. A vantagem do método de Hirota é que ele é mais algébrico que analítico e pode ser facilmente implementado para produzir resultados mais rapidamente. Para implementá-lo, notamos que na equação de $\mathrm{KdV}$, para que tenhamos um balanço entre todos os termos dominantes, introduzimos uma tranformação para novas variáveis dependentes $w=\nu \log (F)$, com grau 0 ,

$$
u=w_{x x}=\nu \partial_{x x} \log (F),
$$

tal que a equação de movimento pode ser escrita como

$$
\left(w_{x t}+\frac{A}{2} w_{x x}^{2}+B w_{x x x x}\right)_{x}=0,
$$

que pode ser integrada de modo que a constante de integração se anule, pois os campos comportam-se como $w \rightarrow 0$ conforme $|x| \rightarrow \infty$. Isso resulta numa equação que tem grau quatro em $F$, com a seguinte estrutura, se fixarmos $\nu=12 \frac{B}{A}$,

$F F_{x t}-F_{x} F_{t}+B\left(3 F_{x x}^{2}-4 F_{x} F_{x x x}+F F_{x x x x}\right)=0$. 
Tal equação pode ser apresentada de uma maneira mais compacta e conveniente,

$$
\left(D_{x} D_{t}+B D_{x}^{4}\right) F \cdot F=0
$$

em termos do chamado operador $D$ de Hirota, definido por

$$
e^{\delta D_{x}} f(x)=f(x+\delta)
$$

de forma que

$$
f(x+\delta) g(x-\delta)=\sum_{n=0}^{\infty} \frac{\delta^{n}}{n !}\left(D_{x}^{n} f(x) \cdot g(x)\right),
$$

onde

$$
D_{x}^{n} f \cdot g=\left.\left(\partial_{x_{1}}-\partial_{x_{2}}\right)^{n} f\left(x_{1}\right) g\left(x_{2}\right)\right|_{x_{2}=x_{1}=x} .
$$

Percebe-se que $D$ opera num produto de duas funções de forma parecida com o operador de Leibnitz, diferindo apenas por um sinal relativo, por exemplo,

$$
\begin{aligned}
D_{x}(f \cdot g) & =f_{x} g-f g_{x} \\
D_{x} D_{t}(f \cdot g) & =f g_{x t}-f_{x} g_{t}-f_{t} g_{x}+f g_{x t} .
\end{aligned}
$$

A equação (21), bilinear em $F$ e escrita em termos do operador de Hirota, denota a forma bilinear de Hirota para a equação de KdV. Sua vantagem é que permite construir soluções por meio de uma expansão do tipo

$$
F(x, t)=f_{0}(x, t)+\epsilon f_{1}(x, t)+\epsilon^{2} f_{2}(x, t)+\ldots,
$$

determinando as soluções $f_{i}(x, t)$ ordem a ordem no parâmetro $\epsilon$.

\subsection{Solução para um sóliton}

A partir da equação de KdV na forma bilinear (21), com a seguinte forma geral

$$
P(D) F \cdot F=0,
$$

para um polinômio $P$ par qualquer dos operadores de Hirota (os termos ímpares se cancelam devido a antissimetria de $D$ ), podemos construir suas soluções por uma expansão em um parâmetro $\epsilon$.

Substituindo tal expansão (22) na equação (23) obtemos

$$
P(D)\left(f_{0} \cdot f_{0}+\epsilon\left(f_{0} \cdot f_{1}+f_{1} \cdot f_{0}\right)+\cdots\right)=0,
$$

e os termos de ordem $\epsilon^{0}$ desaparecem quando $f_{0}(x, t)=$ 1. De fato, sabemos que $u=0$ é uma solução para a
KdV, no vácuo. Para os termos de ordem $\epsilon$ notamos que

$$
f_{1}(x, t)=\exp \left[\sqrt{\frac{v}{B}}(x-v t)\right]
$$

é solução e que para ordens mais elevadas podemos tomar $f_{j}(x, t)=0$, no caso da solução de apenas um sóliton.

Nesse ponto, então, a expressão 20 proporciona

$$
w(x, t)=12 \frac{B}{A} \log \left(1+e^{\sqrt{\frac{v}{B}}(x-v t)}\right)
$$

e obtemos, finalmente a conhecida solução de 1 sóliton para a equação de $\mathrm{KdV}$,

$$
u(x, t)=\frac{3 v}{A} \operatorname{sech}^{2}\left[\frac{1}{2} \sqrt{\frac{v}{B}}(x-v t)\right],
$$

cuja representação gráfica pode ser vista na Figura 5 para diferentes valores de $A$ e $B$.

A partir desta solução 25 mostramos, em seguida, como os valores das constantes $A$, referente ao termo não linear, e $B$, associado ao termo dispersivo da equação de $\mathrm{KdV}$, afetam a forma da
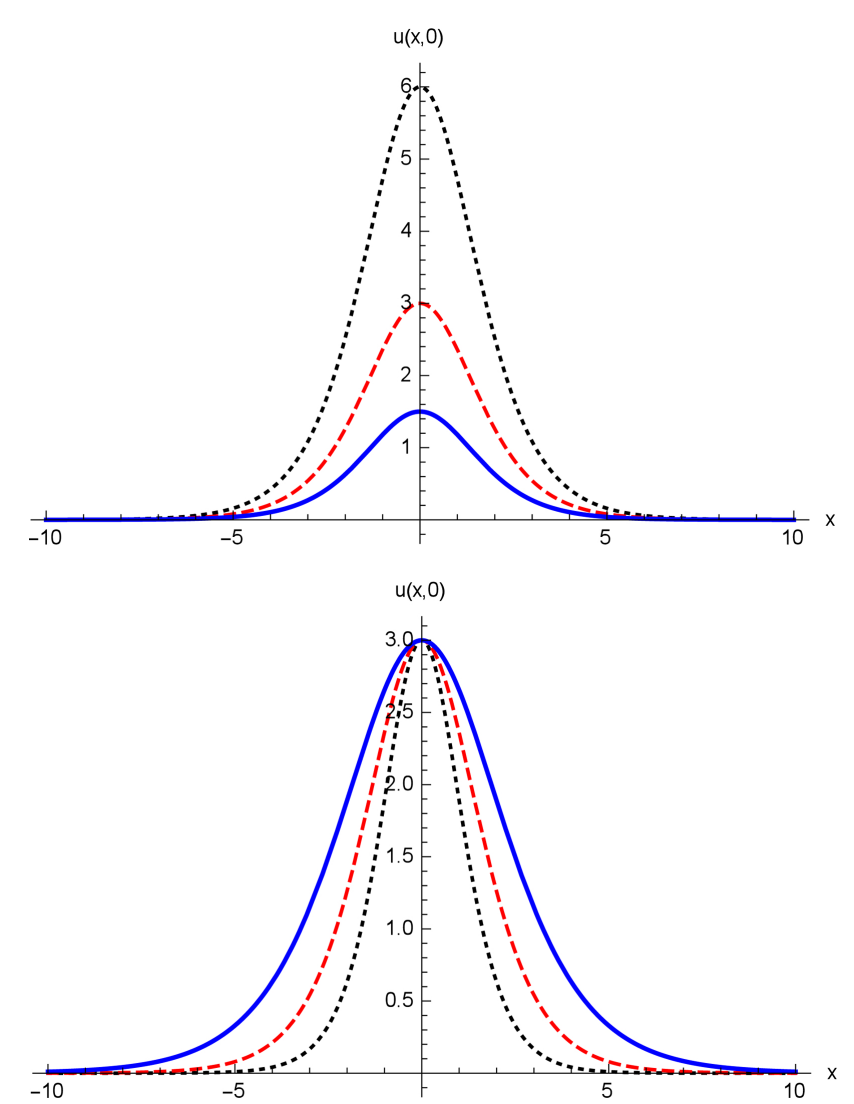

Figura 5: Soluções da KdV para diferentes valores de $A$ (em cima) e $B$ (em baixo). Em linhas contínuas, tracejadas e pontilhadas mostramos esses parâmetros com valores iguais a $0.5,1.0$ e 2.0 , respectivamente. 
sua solução, como visto na Figura (5). Percebemos que, a medida que diminuímos o termo dispersivo, a solução da KdV fica mais estreita (na Figura, à esquerda) e em oposição, quando diminuímos o termo de não linearidade (na Figura, à direita) a solução se torna mais alargada. O importante é que a não linearidade compensa a dispersão criando uma onda que matem sua forma preservada.

Abaixo, na Figura 6, apresentamos a evolução temporal da equação de KdV. Percebemos, na Figura da esquerda, que sua forma permanece inalterada a medida que o tempo passa, isso se deve, à combinação singular entre os termos dispersivo e

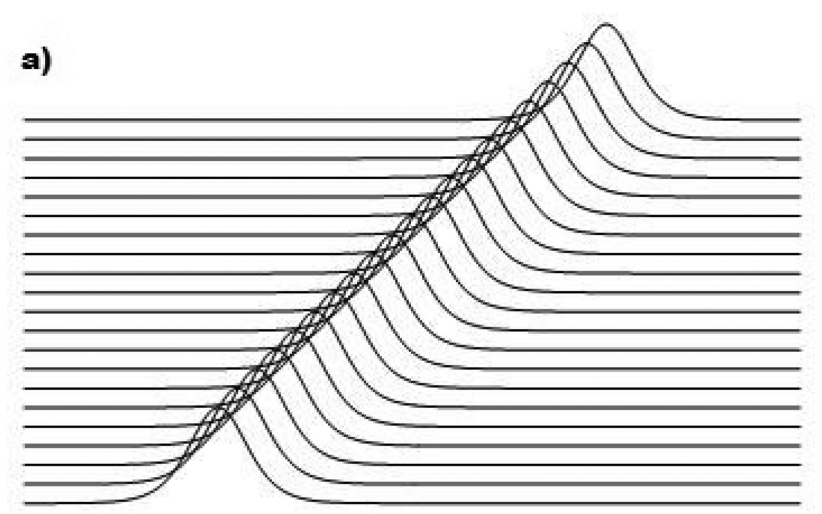

b)
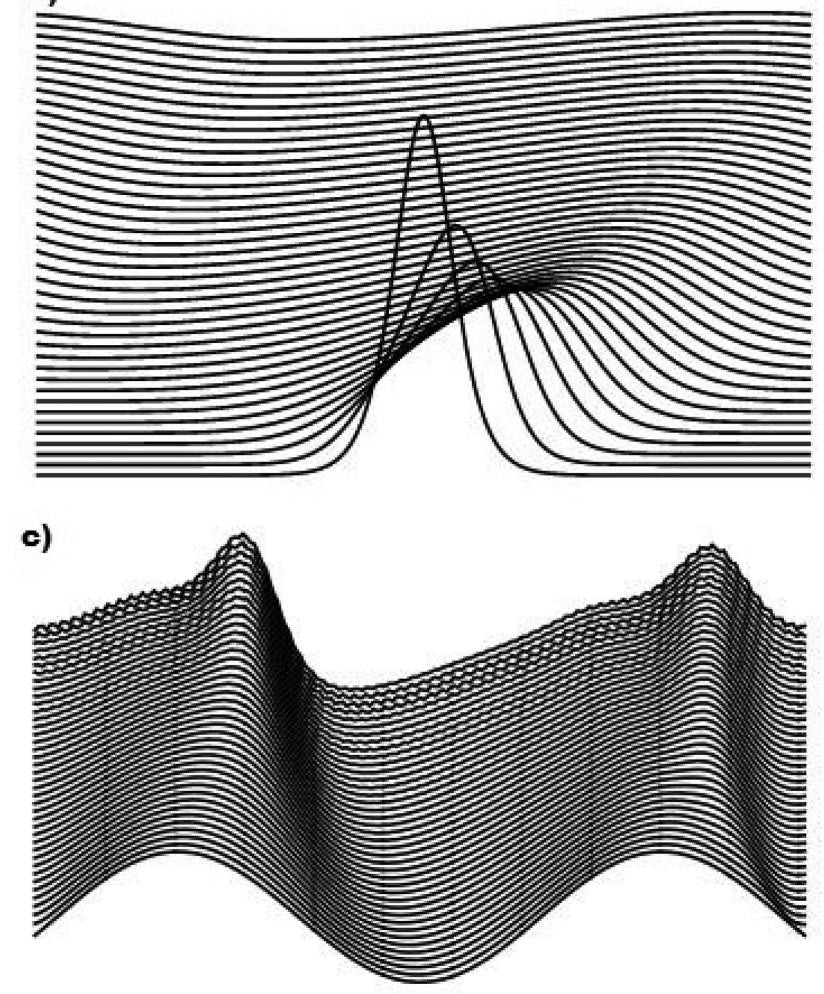

Figura 6: a) Evolução temporal da solução da eq. de KdV. b) Evolução temporal da equação de KdV modificada. c) evolução de um cosseno como condição inical da KdV. não linear da equação de KdV. Introduzindo uma pequena modificação na discretização da equação que quebra a integrabilidade do problema numérico e mostramos o resultado na Figura ao centro. Por fim, apresentamos a evolucão da equação de KdV utilizando como condição incial uma função senoidal, à direita. Percebemos que essa solução não é estável sob a evolução temporal de Korteweg e de Vries, não correspondendo a uma solução solitônica. De fato, dentre as infinitas possibilidades para as condições iniciais apenas uma classe muito restrita comportar-se-á como sólitons.

\subsection{O parentesco entre as equações de KdV e Sine-Gordon}

Tendo visto que a equação de KdV pode ser transformada na equação de Toda e que esta é intimamente ligada à equação de sine-Gordon, aqui buscaremos relacionar mais diretamente as equações de $\mathrm{KdV}$ e sine-Gordon. Note que substituindo

$$
u=v^{2} \pm i \sqrt{\frac{6 B}{A}} v_{x}
$$

na equação de $\mathrm{KdV}$,

$$
u_{t}+A u u_{x}+B u_{x x x}=0,
$$

obtemos

$$
\left(2 v \pm i \sqrt{\frac{6 B}{A}} \partial_{x}\right)\left(v_{t}+A v^{2} v_{x}+B v_{x x x}\right)=0
$$

sendo que a equação

$$
v_{t}+A v^{2} v_{x}+B v_{x x x}=0
$$

é uma equação de KdV modificada denotada por Focusing Miura [23]. Logo, se $v$ satisfizer a equação de KdV modificada então $u$ dada em (26) é uma solução para a equação de KdV original.

Usando a transformação

$$
\begin{aligned}
v & =\frac{\beta}{2} \phi_{x}, \\
u & =\frac{\beta^{2}}{4} \phi_{x}^{2} \pm i \sqrt{\frac{6 B}{A}} \frac{\beta}{2} \phi_{x x}
\end{aligned}
$$

na equação de KdV modificada obtemos

$$
\left(\phi_{t}+\frac{A \beta^{2}}{12} \phi_{x}^{3}+B \phi_{x x x}\right)_{x}=0
$$

Revista Brasileira de Ensino de Física, vol. 39, nº 1, e1307, 2017 
Notamos que uma solução para a equação acima é dada por

$$
\phi(x, t)=\frac{4}{\beta} \tanh ^{-1}\left[\rho e^{\mu\left(\lambda x-\frac{t}{\lambda}\right)}\right]
$$

desde que $A=-6 B, B=\frac{1}{\mu^{2} \lambda^{4}}$ e $\lambda=e^{\chi}$. O curioso deste resultado é que o campo $\phi(x, t)$ acima é solução para a equação de Sinh-Gordon, que se relaciona com o Sine-Gordon por meio da transformação $\beta \rightarrow i \beta$. Na verdade, em [24] os autores mostram que as equações de KdV (modificada) e Sine-Gordon possuem famílias equivalentes de integrais de movimento.

\section{Conclusões e Perspectivas}

Neste trabalho, revisamos alguns dos mais paradigmáticos sistemas não lineares e desvendamos algumas de suas supreendentes interconexões, num contexto acessível a estudantes de graduação, com algum domínio de Mecânica Clássica e Equações Diferenciais. Os problemas de interesse, descritos matematicamente pelas equações de sine-Gordon, Toda e de Kortweg e de Vries (KdV), generalizam modelos físicos bastante conhecidos como o pêndulo simples, o sistema massa-mola e as ondas lineares, respectivamente e oferecem uma boa oportunidade para introduzir fenômenos não-lineares a estudantes de graduação. Após discutirmos as diferenças decorrentes da presença de não-linearidades nos modelos, esclarecemos como os sistemas apresentados relacionam-se entre si, indicando a existência de uma família de equações que compartilham propriedades de integrabilidade.

Procuramos aqui fornecer uma boa fonte da literatura clássica sobre sólitons e sistemas integráveis, mas também incluir trabalhos modernos em dinâmica não-linear, um universo abrangente, com interessantes aplicações. Material em língua portuguesa que trate fenômenos não-lineares e sólitons pode ser encontrado, como em 25[29], mas não em volume compatível com sua importância e ocorrendo predominantemente na forma de dissertações e teses,por exemplo 30 33. Não obstante, vale destacar que a comunidade científica brasileira tem sido bastante ativa nesta área, inserindo-a em universo bastante abrangente que inclui desde a física de plasmas [34, mecânica estatística, 35 39 e.g., equações diferenciais [40 43, física matemática [4-47], altas energias 48, etc. Logo, em meio a tantas ocorrências, reforçamos a ideia de que os sistemas não-lineares correspondem a uma área importante e ativa, merecendo atenção por parte dos estudantes.

\section{Agradecimentos}

Os autores agradecem à Coordenação de Aperfeiçoamento de Pessoal de Nível Superior (CAPES) pelo apoio recebido para o desenvolvimento deste trabalho.

\section{Referências}

[1] E. Noether, Nachr. D. Konig. Gesellsch. D. Wiss. Zu Göttingen, Math-phys. Klasse 1918, 235 (1918).

[2] H. Goldstein, C. Poole and J. Safko, Classical Mechanic (Addison-Wesley, Upper Saddle River, 1980), $3^{\text {a }}$ ed.

[3] A. Das, Integrable Models - Lecture Notes in Physics (World Scientific, Cingapura, 1989).

[4] O. Babelon, D. Bernard and M. Talon, Introduction to Classical Integrable Systems (Cambridge University Press, Cambridge, 2003).

[5] D.J. Korteweg and G. De Vries, Philosophical Magazine 39, 422 (1895).

[6] N.J. Zabusky and M.D. Kruskal, Phys. Rev. Lett. 15, 240 (1965).

[7] P. Lax, Comm. Pure Applied Math. 21, 467 (1968).

[8] E. Bour, J. Ecole Imperiale Polytechnique 19, 1 (1862).

[9] R. Rajaraman, Solitons and Instantons: An Introduction to Solitons and Instantons in Quantum Field Theory (North-Holland Personal Library, NorthHolland, 1989), pp. 34-45.

[10] R.Y. Chiao, E. Garmire and C.H. Townes, Phys. Rev. Lett. 13, 479 (1964).

[11] D.K. Campbell, Nonlinear Science from Paradigms to Practicalities, disponível em http://library. lanl.gov/cgi-bin/getfile?00285753.pdf.

[12] M. Toda, Theory of Nonlinear Lattice (SpringerVerlag, Berlin, 1989).

[13] J. Boussinesq, l'Acad. des Sci. Inst. Nat. France XXIII, 1 (1877).

[14] L.A. Dickey, Soliton Equations and Hamiltonian Systems, Advanced Series in Mathematical Physics: Volume 12 (World Scientific, Singapore, 1991).

[15] P.G. Drazin, Solitons, London Mathematical Society Lecture Note Series 85 ( Cambridge University Press, Cambridge, 1983).

[16] J.S. Russell, in: Report of the Fourteenth Meeting of the British Association for the Advancement of Science(John Murray. London, 1844).

[17] A. Chodos, E. Hadjimicael and C. Tze, Solitons in Nuclear and Elementary Particle Physics (World Scientific Publishing, Singapore, 1985).

[18] T. Dauxois and M. Peyrard Physics of Solitons (Cambridge University Press, Cambridge, 2006). 
[19] L. Debnath, Nonlinear Partial Differential Equations for Scientists and Engineers (Birkhauser, Basel, 2012).

[20] E. Fermi, J. Pasta and S. Ulam, Enrico Fermi Collected Papers (The University of Chicago Press, Chicago, 1965), v. 2, p. 978-988.

[21] M. Toda, Nonlinear Waves and Solitons (KTK Scientific Publishers, Tokyo, 1989).

[22] J. Hietarinta, Physics AU, 15, 31 (2005).

[23] R.M. Miura, C.S. Gardner and M.D. Kruskal, J. Mathematical Phys. 9, 1204 (1968).

[24] Ryu Sasaki and Itaru Yamanaka, Advanced studies in pure mathematics 16, 271 (1987).

[25] F. Chalub and J.P. Zubelli, Solitons: Na Crista da Onda por mais de 100 Anos (Inst. de Matematica Pura e Aplicada, Rio de Janeiro, 2001).

[26] M.A. Savi, Dinâmica Não-Linear e Caos (Editora E-papers, Rio de Janeiro, 2006).

[27] M.S.D. Cattani and J.M.F. Bassalo, Solitrons Hidrodinâmicos de Korteweg-de Vries, Publicação IFUSP 1676 (IFUSP, São Paulo, 2013).

[28] J.L. Cardoso Jr., Solitons Revista Brasileira de Física 10, 695 (1980).

[29] N. Carlin, E.M. Szanto, W.A. Seale, F.O. Jorge, F.A. Souza, I.H. Bechtold and L.R. Gasques, Revista Brasileira de Ensino de Física 27, 225 (2005).

[30] E.E. Leite, Aspectos Não-Perturbativos e Sólitons em Teorias de Campos Integráveis. Tese de Doutorado, IFT-Unesp, 2002.

[31] V.T. Santana, Sólitons e Teorias Não Lineares Integráveis. Tese de Doutorado, Universidade de São Paulo, 2009.

[32] L.E. Arroyo Meza, Equação de Schrodinger Não Linear com Coeficientes Modulados. Tese de Doutorado, IFT-Unesp, 2015.

[33] R.A.C Correa, Solitons e Oscillons em cenários com violações da simetria de Lorentz. Tese de Doutorado, IFT-Unesp, 2014.

[34] A. Bonatto, R.P. Nunes, C. Bonatto, R. Pakter, S.R. Lopes and F.B. Rizatto, Physica A: Statistical Mechanics and its Applications 404, 332 (2014).

[35] E. Abdalla, M.C.B. Abdalla and K.D. Rothe, NonPerturbative Methods in 2 Dimensional Quantum Field Theory (World Scientific, Singapore, 1991), 2nd ed.

[36] C.G. Rodrigues, A.A.P. Silva, A.R.V. Silva, J.G. Ramos and R. LUZZI, Brazilian Journal of Physics 40, 63 (2010).

[37] L.A.N. de Paula, Brazilian Journal of Physics 39, 711 (2009).

[38] R.A.S. Silva, E. Drigo Filho, and J.R. Ruggiero, Brazilian Journal of Physics 38, 362 (2008).

[39] P.E.G. Assis, arXiv:1608.00845[cond-mat.statmech].

[40] J. Angulo and F.M.A. Natali, Advances in Nonlinear Analysis 3, 95 (2014).
[41] R.J. Iorio Jr. in: Nonlinear Equations: Methods, Models and Applications (Birkhauser, Basel, 2003), p. 163-179.

[42] R. Cote, C. Munoz, D. Pilod and G. Simpson, Archive for Rational Mechanics and Analysis 220, 639 (2016).

[43] C.E. Kenig and D. Pilod, Advances in Differential Equations 21801 (2016).

[44] J.C. Brunelli, Brazilian Journal of Physics 30, 455 (2000).

[45] A. Belendez, C. Pascual, T. Mendez, T. Belendez, and C. Neipp, Revista Brasileira de Ensino de Física 29, 645 (2007).

[46] C.A. Linhares and H.P. Oliveira, Brazilian Journal of Physics 37, 368 (2007).

[47] P.E.G. Assis and A. Fring, PRAMANA Indian Academy of Sciences 74, 857 (2010).

[48] M. Gleiser, Brazilian Journal of Physics 36, 1150 (2006).

[49] P.E.G. Assis and L.A. Ferreira, Nuclear Physics B 800, 409 (2008). 